\title{
PRK versus LASIK para correção de miopia baixa e moderada
}

\author{
PRK versus LASIK for correction of low and moderate myopia
}

Adriana dos Santos Forseto (1)

Regina Aidar Menon Nosé (1)

Walton Nosé $(1,2,3)$

\section{RESUMO}

Objetivo: Comparar, por meio de um estudo prospectivo e bilateral randomizado, a eficácia, segurança e previsibilidade das técnicas de ceratectomia fotorrefrativa (PRK) e "excimer laser in situ keratomileusis" (LASIK) para o tratamento da miopia baixa e moderada.

Métodos: Foram incluídos dezessete pacientes $(34$ olhos) com equivalente esférico (EE) refracional entre -2,00 e-6,00 dioptrias (D). Cada paciente foi submetido a PRK em um olho e LASIK no outro. Ambos os olhos foram tratados no mesmo dia pelo mesmo cirurgião, com o excimer laser VISX 20/20B e o microceratótomo automatizado da Chiron.

Resultados: No pré-operatório, a média do EE refracional foi semelhante nos dois grupos $(-3,57 \pm 1,17 D$ e $-3,71 \pm 0,98 D$, para PRK e LASIK, respectivamente). Dor pós-operatória foi referida somente nos casos de PRK (8 olhos: 47,1\%). O seguimento pós-operatório médio foi de $14 \pm 5$ meses (6 a 24) para o grupo de PRK, e $14 \pm 6$ meses (3 a 24) para o de LASIK. A média do erro refrativo residual foi semelhante nos dois grupos $(-0,34 \pm 0,45 D$ e $-0,29 \pm 0,52 D$, para PRK e LASIK, respectivamente). No primeiro dia pós-tratamento, 15 olhos (100\%) do grupo de LASIK e $2(\mathbf{1 3 , 3 \% )}$ de PRK apresentavam acuidade visual sem correção $(\mathrm{AV} s / \mathrm{c}) \geq 20 / 40(n=15)$. Ao último exame, 15 olhos $(88,2 \%)$ de LASIK contra $11(64,7 \%)$ de PRK atingiram AV s/c $\geq 20 / 20(n=17)$. Não houve perda da melhor acuidade visual corrigida.

Conclusão: Neste estudo, tanto o PRK quanto o LASIK mostraram-se ser tecnicamente seguros, eficazes e previsíveis para a correção da miopia baixa e moderada. A técnica de LASIK foi a preferida na análise subjetiva dos pacientes, pela recuperação visual mais precoce associada a menor dor no pós-operatório.

Palavras-chave: Miopia; Excimer laser; LASIK; Cirurgia refrativa.

\section{INTRODUÇÃO}

As técnicas cirúrgicas para a correção da baixa miopia geralmente são realizadas na córnea. A possibilidade de se remover áreas específicas e de espessura pré-determinada da córnea, através da ablação do tecido com excimer laser, foi demonstrada pela primeira vez em 1983 por Trokel et al. ${ }^{1}$ Posteriormente, o primeiro olho com visão foi tratado com sucesso pela técnica de ceratectomia fotorrefrativa (PRK) ${ }^{2}$. Poucos anos após o seu uso na remodelação da superfície corneana, o excimer laser foi aplicado na cirurgia refrativa lamelar ${ }^{3}$. 
A técnica de LASIK (laser in situ keratomileusis), inicialmente reservada para o tratamento da alta miopia, ${ }^{4}$ vem se difundindo cada vez mais em nosso meio, principalmente pelo impacto da recuperação visual causado no período pósoperatório, além da redução da dor e desconforto dos pacientes ${ }^{5}$. Por estes motivos, alguns autores preconizam o seu uso também para o tratamento de miopia baixa e moderada ${ }^{5-8}$.

Este estudo prospectivo e bilateral randomizado tem como objetivo comparar a eficácia, segurança e previsibilidade das técnicas de PRK e LASIK para o tratamento de miopia entre $-2,00$ e $-6,00$ dioptrias (D).

\section{MATERIAL E MÉTODOS}

Vinte pacientes ( 40 olhos) com equivalente esférico (EE) refracional entre -2,00 e -6,00 dioptrias foram incluídos neste estudo. Cada paciente foi aleatoriamente tratado no mesmo dia com PRK em um olho e LASIK no olho contra-lateral, sendo o procedimento realizado pelo mesmo cirurgião (WN).

Ambas as técnicas já foram aprovadas para uso clínico no Brasil pelo Conselho Federal de Medicina e Conselho Brasileiro de Oftalmologia. Desta forma, obteve-se termo de consentimento assinado em todos os casos, após explicação detalhada dos procedimentos cirúrgicos, seus riscos e outras opções para a correção de suas ametropias.

A avaliação pré-operatória consistiu de exame oftalmológico completo, incluindo ainda topografia computadorizada da córnea (Alcon Eye Map EH-290) e paquimetria ultrassônica (Ultrasonic Pachymeter Alcon). Os pacientes foram orientados a suspender o uso de suas lentes de contato por período mínimo de 1 semana. Outros critérios de inclusão foram os seguintes: estabilidade refracional por período mínimo de 1 ano, acuidade visual com correção $(\mathrm{AVc} / \mathrm{c})$ préoperatória melhor ou igual a 20/40 em ambos os olhos, diferença de $\mathrm{EE}$ entre os dois olhos igual ou inferior a 1,0D, astigmatismo menor ou igual a $1,75 \mathrm{DC}$.

Todas as cirurgias foram realizadas sob anestesia tópica (cloridrato de proximetacaína, Anestalcon ${ }^{\circledR}$ ). A fotoablação corneana foi feita com o aparelho de excimer laser VISX 20/ 20B (versão 4,02c), que trabalha com fluência de $160 \mathrm{~mJ} / \mathrm{cm}^{2}$ e taxa de repetição de $6 \mathrm{~Hz}$. Para miopias de até $-6,0 \mathrm{D}$ este aparelho pode realizar apenas uma zona óptica de $6 \mathrm{~mm}$ (programa multizona); ou zonas de 4,5 a $6,0 \mathrm{~mm}$, com incrementos de $0,5 \mathrm{~mm}$ (programa multipass/multizona). $\mathrm{O}$ astigmatismo, quando presente, foi corrigido pelo método elíptico ${ }^{9}$.

Para a realização do PRK, o cirurgião marcou e removeu cuidadosamente o epitélio em torno de $6,5 \mathrm{~mm}$ de diâmetro da região central. A fotoablação foi centralizada na pupila de entrada do paciente, tendo sido utilizado o programa multipass/multizona. Após a cirurgia colocou-se uma lente de contato terapêutica, sendo prescrito colírios de tobramicina 0,3\% (Tobrex ${ }^{\circledR}: 6 / 6$ horas até epitelização), diclofenaco sódico (Still ${ }^{\circledR}: 8 / 8 \mathrm{~h}$ por 2 dias) e acetato de fluormetolona $0,1 \%$ (Florate $^{\circledR}: 6 / 6 \mathrm{~h}$ por 15 dias, regredindo-se gradualmente até o término do terceiro mês pós-operatório).
A preparação do disco corneano e leito estromal no LASIK foi realizada com o microceratótomo Automated Corneal Shaper (Chiron Ophthalmics ${ }^{\circledR}$ ), com plataforma de $160 \mu \mathrm{m}$ e diâmetro de $8,5 \mathrm{~mm}$. Utilizamos nestes casos o programa multizona. No final do procedimento, o disco foi reposicionado, sem a necessidade de oclusão ou lentes de contato terapêuticas, tendo sido prescrito colírio de tobramicina $0,3 \%$ e dexametasona $0,1 \%\left(\right.$ Tobradex $^{\circledR}: 4 / 4 \mathrm{~h}$ por 10 dias).

As visitas pós-operatórias foram agendadas para os dias 1, 3 a 5,15; e meses $1,3,6,12$ e 24 , quando foram realizados exames oftalmológicos completos. Possíveis achados biomicroscópicos de opacidade corneana ("haze") foram quantificados de 0 a 4 +: 0 (sem haze), $0,5+$ (traços: padrão granular de difícil detecção), 1+ (traços: leve padrão granular acinzentado), 2+ (leve: padrão reticular acinzentado facilmente visível), 3+ (moderado: placa densa acinzentada); 4+ (severo: densa cicatriz esbranquiçada). A presença de dor no pós-operatório imediato também foi avaliada, sendo classificada subjetivamente em leve, moderada e severa.

No exame de 12 meses, a satisfação e preferência dos pacientes foram avaliadas através de um questionário. Inicialmente eles foram questionados sobre qual técnica cirúrgica havia sido utilizada em cada olho (se lembravam ou não). $\mathrm{Na}$ seqüência, perguntava-se sobre a preferência em relação ao per e pós-operatório, presença de dor, se operariam novamente ou se indicariam o procedimento a um amigo. A análise subjetiva sobre a acuidade e qualidade de visão em ambos os olhos foi feita com a seguinte pergunta: "Em termos gerais, a visão de qual olho você prefere hoje: direito, esquerdo ou indiferente?". Caso houvesse diferença, anotávamos aquele considerado como o de melhor visão e então correlacionávamos com a técnica empregada naquele olho.

As variáveis quantitativas foram representadas por média, desvio padrão (DP), mediana, valores mínimo e máximo. As variáveis qualitativas foram representadas por freqüência absoluta (n) e relativa (\%). Foram aplicados testes paramétricos e não paramétricos devido à natureza dos dados (grande variabilidade). Adotou-se o nível de significância de 0,05 ( $\alpha=$ $5 \%$ ). Níveis descritivos (P) inferiores a esse valor foram considerados e representados por *.

\section{RESULTADOS}

Todas as cirurgias foram realizadas sem intercorrências não tendo sido observadas complicações per-operatórias $(\mathrm{n}=40)$. Três pacientes não retornaram aos exames pós- operatórios propostos, portanto os resultados apresentados referem-se aos dados de 17 pacientes (34 olhos), com idade média de $29 \pm 7$ anos (variando de 20 a 48 anos). Destes 17 pacientes, $15(88,2 \%)$ eram do sexo feminino. Todos apresentaram seguimento mínimo de 12 meses, com exceção de um, devido a hipocorreção e indicação precoce de retratamento bilateral no terceiro e sexto mês. Portanto, o seguimento pós- 
operatório médio em meses foi de $14 \pm 5$ ( 6 a 24 meses) para o grupo de PRK, e $14 \pm 6$ ( 3 a 24 meses) para o grupo de LASIK.

A profundidade de ablação variou entre 20 e $60 \mu \mathrm{m}$ (média: $42,18 \pm 12,05 \mu \mathrm{m}$ ) para o grupo de PRK, e entre 24 a $70 \mu \mathrm{m}$ (média: $49,41 \pm 12,00 \mu \mathrm{m})$ para o de LASIK. $(\mathrm{P}=0,195$, Mann-Whitney).

A epitelização da córnea, nos olhos submetidos a PRK, ocorreu entre 3 e 7 dias (média: 4,73 $\pm 1,09$ dias), época em que foi retirada a lente de contato terapêutica.

Trinta e três olhos $(97,05 \%)$ apresentavam acuidade visual sem correção (AV s/c) no pré-operatório pior ou igual a 20/ 100 , sendo que apenas um dos casos apresentava visão de 20/ 70. Ao último exame, o grupo de LASIK mostrou uma maior porcentagem de olhos com visão sem correção melhor ou igual a 20/20: 15 olhos $(88,2 \%)$ contra $11(64,7 \%)$ para o grupo de PRK. Entretanto, esta diferença não foi considerada estatisticamente significante $(\mathrm{P}=0,225)$. Por sua vez, a porcentagem de olhos com AV s/c $\geq 20 / 40$ foi bastante semelhante nos dois grupos: $14(82,4 \%)$ e 15 olhos $(88,2 \%)$ para PRK e LASIK respectivamente. $(\mathrm{P}=1,000)$

A recuperação da acuidade visual também ocorreu de forma mais rápida no grupo de LASIK, sendo que no primeiro dia pósoperatório 12 olhos (80\%) apresentavam AV s/c $\geq 20 / 25$, e 15 $(100 \%) \geq 20 / 40(n=15)$. Nesta ocasião, somente 2 casos $(13,3 \%)$ do grupo de PRK apresentavam AV s/c $\geq 20 / 40$ (Gráfico 1).

As alterações ceratométricas e do astigmatismo refracional podem ser melhor analisadas nas tabelas 1 e 2 .

A média do EE no pré-operatório foi de $-3,57 \pm 1,17 \mathrm{D}$ (variando de $-5,25$ a $-1,62 \mathrm{D})$ e $-3,71 \pm 0,98 \mathrm{D}(-5,27$ a $-1,88 \mathrm{D})$ para os grupos de PRK e LASIK respectivamente. Não foi observada diferença estatisticamente significante entre a refração estática pré-operatória para estes dois grupos. $(\mathrm{P}=$ 0,666, Mann-Whitney).

Ao último exame, os dois grupos apresentaram uma redução estatisticamente significante na refração em comparação aos valores pré-operatórios ( $\mathrm{P}<0,001^{*}$, Wilcoxon). $\mathrm{O}$ grupo de $\mathrm{PRK}$ ficou com EE entre $0,25 \mathrm{D}$ e $-1,5 \mathrm{D}$ (média: $-0,34 \pm 0,45 \mathrm{D}$ ), e o de

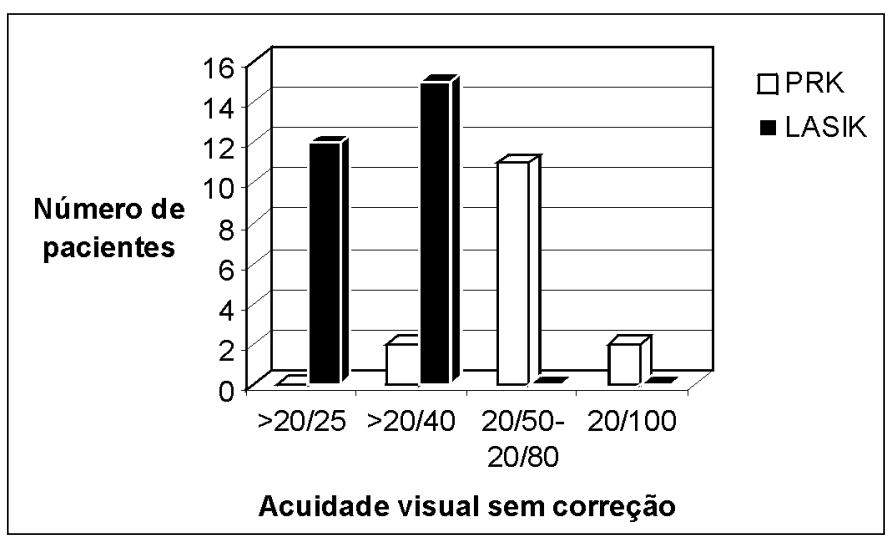

Gráfico 1 - Avaliação da acuidade visual sem correção entre os grupos de PRK e LASIK no primeiro dia pós-operatório $(n=15)$

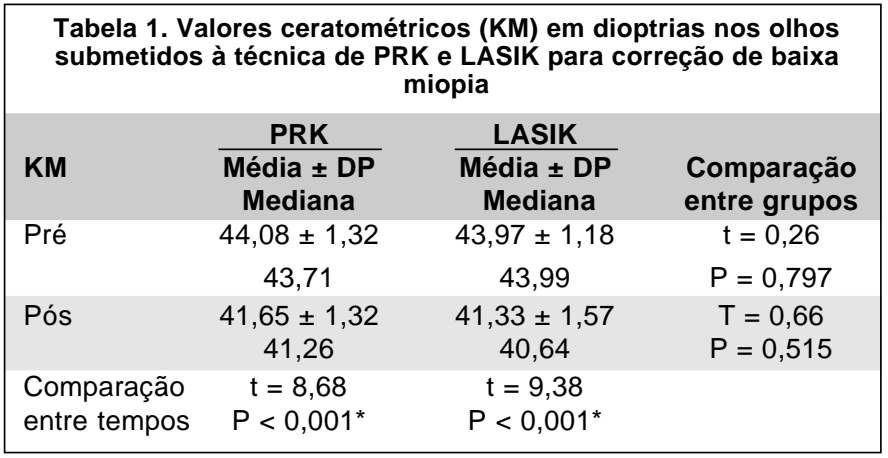

\begin{tabular}{|lccc|}
\hline \multicolumn{4}{|c|}{$\begin{array}{c}\text { Tabela 2. Astigmatismo refracional em dioptrias cilíndricas (DC) } \\
\text { nos olhos submetidos à técnica de PRK e LASIK para correção } \\
\text { de baixa miopia }\end{array}$} \\
Astigmatismo & $\begin{array}{c}\text { PRK } \\
\text { Média } \pm \text { D.P. } \\
\text { Mediana }\end{array}$ & $\begin{array}{c}\text { Média } \pm \text { D.P. } \\
\text { Mediana }\end{array}$ & $\begin{array}{c}\text { Comparação } \\
\text { entre grupos }\end{array}$ \\
Pré & $-0,60 \pm 0,49$ & $-0,62 \pm 0,57$ & $U=144,0$ \\
& $-0,50$ & $-0,50$ & $P=0,986$ \\
Pós & $-0,24 \pm 0,27$ & $-0,25 \pm 0,29$ & $U=141,5$ \\
& 0,00 & 0,00 & $P=0,894$ \\
Comparação & $z=2,51$ & $z=2,53$ & \\
entre tempos & $P=0,012^{*}$ & $P=0,012^{*}$ & \\
\hline
\end{tabular}

LASIK entre 0,25D e -1,88D (média: -0,29 $\pm 0,52 \mathrm{D}$ ). Não houve entretanto diferença entre eles. ( $\mathrm{P}=0,443$, Mann-Whitney)

Quinze olhos $(88,2 \%)$ do grupo de LASIK e $14(82,4 \%)$ de PRK ficaram com EE refracional dentro da faixa de $\pm 0,50 \mathrm{D}$ da emetropia. $(\mathrm{P}=1,000)$ Também não foi observada diferença em relação ao número de olhos entre $\pm 1,00 \mathrm{D}(15$ ou $88,2 \%)$ e $\pm 2,00 \mathrm{D}$ (17 ou $100 \%)$ para os dois grupos. $(\mathrm{P}=1,000)$ Dois pacientes ( 4 olhos) ficaram fora da faixa entre $\pm 1,00 \mathrm{D}$, tendo sido indicado retratamento precoce para um deles por insatisfação quanto ao resultado final. O retratamento foi realizado com a técnica de LASIK em ambos os olhos.

Não houve alteração na melhor acuidade visual corrigida no pós-operatório, tendo sido mantido portanto, o valor de 20/20 em todos os casos.

Não foram observadas diferenças entre as médias das medidas da pressão intra-ocular pré e última consulta pós tratamento nos dois grupos, ou entre eles. Ao exame de 1 mês pós-operatório, foi receitado colírio beta-bloqueador (maleato de timolol $0,5 \%)$ para um paciente $(5,8 \%)$, somente no olho submetido a PRK, devido a elevação dos níveis pressóricos.

Dor pós-operatória foi referida somente nos casos de PRK, sendo classificada em leve (3 olhos: 17,64\%), moderada (3 olhos: 17,64\%), e severa (2 olhos: 11,76\%). Neste último grupo, a causa foi atribuída à perda da lente de contato terapêutica.

Discreta opacidade cristalineana subcapsular posterior, não previamente constatada, foi observada em 2 pacientes (2 olhos: 5,8\%). Um caso foi submetido a PRK e outro a LASIK. 
Traços de opacidade corneana ("haze": $0,5+$ ) foi observada apenas em $2(11,76 \%)$ dos 17 olhos submetidos a PRK, no $1^{\circ} \mathrm{e} 12^{\circ}$ meses pós-operatório.

Onze $(64,7 \%)$ dos 17 pacientes responderam ao questionário entregue no retorno de 1 ano de cirurgia. Destes, 10 $(90,9 \%)$ recordavam a técnica cirúrgica que havia sido utilizada, apontando corretamente o olho submetido a cada procedimento. Seis pacientes $(54,5 \%)$ não notaram diferença em relação ao intra-operatório entre as 2 técnicas empregadas; $3(27,3 \%)$ preferiram o LASIK e os outros $2(18,2 \%)$ o PRK. Entretanto, todos os pacientes relataram preferência pelo LASIK no período pós-operatório, com exceção de um. Oito $(72,7 \%)$ lembraram que sentiram algum tipo de desconforto ou dor no olho submetido ao PRK, $2(18,2 \%)$ apresentaram esta sensação nos dois olhos, e $1(9,0 \%)$ não sabia referir. Todos se submeteriam a cirurgia novamente ou a indicariam a um amigo. Em termos gerais, seis pacientes $(54,5 \%)$ acharam que não havia diferença entre a sua visão final comparando-se os dois olhos, $3(27,3 \%)$ preferiram o olho do LASIK e 2 $(18,2 \%)$ o do PRK.

\section{DISCUSSÃO}

Apesar da existência de um maior número de pessoas com baixa miopia na população geral quando comparados a indivíduos com miopia alta ou extrema, McCarty et al. demonstraram que a distribuição dos erros refrativos em determinado centro de cirurgia a laser era muito semelhante entre estes 3 grupos ${ }^{10}$. Isto representa uma baixa representação da população com baixa miopia dentre os casos submetidos a cirurgia refrativa. Em termos de potencial mercadológico, parece haver uma deficiência na divulgação de informações e resultados dos procedimentos refrativos para este grupo. Ou talvez estes pacientes, pelo fato de apresentarem uma boa acuidade visual corrigida e não serem extremamente dependentes da correção óptica, sejam mais exigentes quanto ao resultado, segurança e recuperação visual de uma possível cirurgia.

A eficácia e segurança da ceratectomia fotorrefrativa para o tratamento da miopia baixa já foi demonstrada por Hayashi et al. Em seu estudo, $100 \%$ dos olhos com EE préoperatório $\leq-5,00 \mathrm{D}$, atingiram visão não corrigida melhor ou igual a $20 / 40$, e $93 \%$ visão de $20 / 20$ ou melhor ao exame de 9 meses pós- operatório ${ }^{11}$.

A recuperação visual ocorre mais precocemente no LASIK ${ }^{7,12}$, influenciando desta forma o grau de satisfação dos pacientes, e retorno a suas atividades laborativas ${ }^{12}$. Alguns estudos de LASIK para correção da baixa miopia já foram propostos ${ }^{5-8}$. Entretanto, existe uma preocupação em relação ao seu emprego para esta faixa da população, pelo fato de ser uma cirurgia tecnicamente mais difícil e não estar isenta de complicações ${ }^{13}$, além do fator custo, que envolve a disponibilidade de um aparelho de microceratótomo para a confecção do disco corneano.
Assim como no artigo de Maghraby et al., ${ }^{14}$ este estudo controlou uma série de variáveis pelo fato de termos utilizado o mesmo cirurgião operando o mesmo equipamento, nos dois olhos do mesmo paciente durante a mesma sessão cirúrgica, para compararmos 2 procedimentos refrativos diferentes: PRK e LASIK.

Apesar de um pequeno número de casos (17 pacientes; 34 olhos), a bilateralidade fez com que os dois grupos fossem estatisticamente comparáveis, tendo sido incluídos apenas os casos com diferença de EE refracional entre os 2 olhos $\leq 1,00 \mathrm{D}$. A dificuldade de se realizar um estudo prospectivo foi observada pela perda de seguimento em 3 pacientes, que referiam impossibilidade de retornar a todos seguimentos propostos, considerando-os de certa forma desnecessários, já que estavam satisfeitos com o resultado cirúrgico final.

A recuperação da acuidade visual ocorreu mais precocemente no LASIK, onde $80 \%$ dos olhos apresentavam visão sem correção $\geq 20 / 25$ já no primeiro pós-operatório. Salah et al. mostraram um estudo onde o LASIK foi utilizado para correções de miopia de 2,00 a 20,00 dioptrias. ${ }^{7}$ Neste estudo, os pacientes relataram acuidade visual funcional dentro do período de 24 horas após a realização do procedimento, semelhante à nossa experiência clínica.

Ao último exame, o grupo de LASIK também mostrou uma maior porcentagem de olhos com visão sem correção $\geq 20 / 20$ ( $88,2 \%$ contra $64,7 \%$ no PRK), apesar desta diferença não ter sido estatisticamente significante. Estes dados coincidem com a avaliação subjetiva feita pelo questionário dos pacientes, onde em termos gerais, $54,5 \%$ acharam que não havia diferença entre a sua visão final comparando-se os dois olhos, 3 $(27,3 \%)$ preferiram o olho do LASIK e $2(18,2 \%)$ o do PRK. Wang et al. também mostraram em estudo comparativo entre LASIK e PRK para miopias de até 6,00 dioptrias, que pacientes submetidos a LASIK atingiram melhor acuidade visual final sem correção. Neste estudo, ao exame de 1 ano pósoperatório, $83 \%$ dos pacientes operados com a técnica de LASIK e $72 \%$ pela de PRK apresentavam acuidade visual sem correção melhor ou igual a 20/20 ${ }^{12}$.

A média do erro refrativo residual foi semelhante nos dois grupos, o que era esperado pelo fato de termos utilizado o mesmo aparelho de excimer laser e o mesmo cirurgião no mesmo tempo cirúrgico. Não observamos diferenças na correção entre os dois programas de tratamento utilizados (multizona no LASIK e multipass/multizona no PRK). Nossos resultados foram semelhantes ao estudo de Danasoury et al. na correção de miopia entre 2,00 e 5,50 D, pelas técnicas de PRK e LASIK ${ }^{15}$ (Tabela 3).

Lipshitz et al., em estudo comparativo e retrospectivo entre PRK e LASIK, encontraram diferença estatisticamente significante ao analisar o equivalente esférico refracional pósoperatório. Resultados superiores foram obtidos com a técnica de LASIK, o que talvez possa ser explicado pelo fato dos autores terem tratado olhos com ametropias superiores às do nosso estudo (-7,12 D e -7,47 D, em média, para os grupos de PRK e LASIK respectivamente) ${ }^{16}$. 


\begin{tabular}{|c|c|c|c|c|c|c|c|c|}
\hline Autor & Técnica & Olhos & EE pré (D) & EE pós (D) & $E E \pm 1,00 D$ & $E E \pm 0,50 D$ & $\begin{array}{l}\text { AV s/c } \\
\geq 20 / 20\end{array}$ & Tempo (anos) \\
\hline \multirow[t]{2}{*}{$\begin{array}{l}\text { El-Maghraby } \\
\text { et al. (1999) }\end{array}$} & PRK & 33 & $\begin{array}{c}-4,70 \pm \\
1,50\end{array}$ & $\begin{array}{c}-0,10 \pm \\
0,60 \mathrm{D}\end{array}$ & $\begin{array}{c}26 \\
(87 \%)\end{array}$ & $\begin{array}{c}20 \\
(67 \%)\end{array}$ & $\begin{array}{c}10 \\
(36 \%)\end{array}$ & $1-2$ \\
\hline & LASIK & 33 & $\begin{array}{c}-4,80 \pm \\
1,60\end{array}$ & $\begin{array}{c}0,0 \pm \\
0,60 \mathrm{D}\end{array}$ & $\begin{array}{c}27 \\
(90 \%)\end{array}$ & $\begin{array}{c}22 \\
(73 \%)\end{array}$ & $\begin{array}{c}18 \\
(61 \%)\end{array}$ & $1-2$ \\
\hline \multirow[t]{2}{*}{$\begin{array}{l}\text { Danasoury } \\
\text { et al. (1999) }\end{array}$} & & 26 & $\begin{array}{c}-3,23 \pm \\
0,63\end{array}$ & $\begin{array}{l}-0,08 \pm \\
0,38 D\end{array}$ & $\begin{array}{c}24 \\
(100 \%)\end{array}$ & $\begin{array}{c}20 \\
(83 \%)\end{array}$ & $\begin{array}{c}15 \\
(63 \%)\end{array}$ & 1 \\
\hline & LASIK & 26 & $\begin{array}{c}-3,44 \pm \\
0,72\end{array}$ & $\begin{array}{l}-0,14 \pm \\
0,31 \mathrm{D}\end{array}$ & $\begin{array}{c}24 \\
(100 \%)\end{array}$ & $\begin{array}{c}21 \\
(88 \%)\end{array}$ & $\begin{array}{c}19 \\
(79 \%)\end{array}$ & 1 \\
\hline \multirow[t]{2}{*}{ Nosso estudo } & PRK & 17 & $\begin{array}{c}-3,57 \pm \\
1,17\end{array}$ & $\begin{array}{l}-0,34 \pm \\
0,45 D\end{array}$ & $\begin{array}{c}15 \\
(88 \%)\end{array}$ & $\begin{array}{c}14 \\
(82 \%)\end{array}$ & $\begin{array}{c}11 \\
(65 \%)\end{array}$ & 1 \\
\hline & LASIK & 17 & $\begin{array}{c}-3,71 \pm \\
0,98\end{array}$ & $\begin{array}{l}-0,29 \pm \\
0,52 \mathrm{D}\end{array}$ & $\begin{array}{c}15 \\
(88 \%)\end{array}$ & $\begin{array}{c}15 \\
(88 \%)\end{array}$ & $\begin{array}{c}15 \\
(88 \%)\end{array}$ & 1 \\
\hline
\end{tabular}

Dois pacientes (4 olhos) ficaram hipocorrigidos, fato provavelmente explicado por alteração de cicatrização individual ou calibragem do equipamento, e não pela técnica cirúrgica empregada.

A segurança do procedimento pôde ser observada pela manutenção da melhor acuidade visual corrigida nos dois grupos. Apenas um paciente apresentou elevação transitória da pressão intra-ocular no PRK, o que foi controlado com a utilização de colírio hipotensor. A opacidade corneana observada no PRK também foi considerada leve nos dois casos, sem alteração da acuidade visual.

Mesmo 1 ano após o procedimento cirúrgico, $72,7 \%$ dos casos ainda lembravam que sentiram dor no pós-operatório de PRK, o que levou alguns pacientes a contra-indicarem esta técnica aos amigos, dando preferência ao LASIK.

Neste estudo, tanto o PRK quanto o LASIK mostraram-se ser tecnicamente seguros, eficazes e previsíveis para a correção da baixa miopia. A técnica de LASIK foi a preferida na análise subjetiva dos pacientes, pela recuperação visual mais precoce associada a menor dor no pós-operatório.

\section{SUMMARY}

Purpose: To compare, through a prospective, randomized and bilateral study, the effectiveness, safety and predictability of photorefractive keratectomy (PRK) and excimer laser in situ keratomileusis (LASIK) for correction of low and moderate myopia.

Methods: Seventeen patients (34 eyes) with preoperative refractional spherical equivalent (SE) ranging from -2.00 to -6.00 diopters $(D)$ were enrolled in this study. Each patient received PRK in one eye and LASIK in the other. Both eyes were treated by the same surgeon during the same operative session with the excimer laser VISX 20/20B and the Chiron Automated Corneal Shaper microkeratome.

Results: Preoperatively the mean SE was the same for both groups $(-3.57 \pm 1.17 D$ and $-3.71 \pm 0.98 D$, for PRK and LASIK groups, respectively). Pain was reported only by the
PRK cases (8 eyes: $47.1 \%)$. The average follow-up was $14 \pm$ 5 months (6 to 24) for the PRK group, and $14 \pm 6$ months (3 to 24) for the LASIK group. The mean residual refractive error was similar after both procedures $(-0.34 \pm 0.45 D$ and $-0.29 \pm 0.52 D$, for PRK and LASIK, respectively). At 1 day after surgery, 15 LASIK eyes (100\%) and 2 PRK eyes (13.3\%) had an uncorrected visual acuity (UCVA) $\geq 20 / 40$ $(n=15)$. At last follow-up, 15 eyes from the LASIK group (88.2\%) and 11 from the PRK group (64.7\%) reached a UCVA $\geq 20 / 20(n=17)$. The best spectacle corrected visual acuity remained unchanged in all cases.

Conclusion: Both PRK and LASIK were found to be similarly safe, effective and predictable for the correction of low and moderate myopia. Patients preferred LASIK because of fast and painless visual recovery.

Keywords: Myopia; Excimer laser; LASIK; Refractive surgery.

\section{REFERÊNCIAS BIBLIOGRÁFICAS}

1. Trokel SL, Srinivasan R, Braren B. Excimer laser surgery of the cornea. Am J Ophthalmol 1983;96:710-5.

2. McDonald MB, Liu JC, Byrd TJ, Abdelmegeed M, Andrade HA, Klyce SD, Varnell R, Munnerlyn CR, Clapham TN, Kaufman HE. Central photorefractive keratectomy for myopia: partially sighted and normally sighted eyes. Ophthalmol 1991;98:1327-37.

3. Farah SG, Azar DT, Gurdal C, Wong J. Laser in situ keratomileusis: literature review of a developing technique. J Cataract Refract Surg 1998;24:989-1006.

4. Carvalho M, Nascimento E, Chamon W, Alleman N, Campos M, Scarpi MJ. Lasik na correção da alta miopia. Arq Bras Oftal 1997;60(6):570-5.

5. Pirzada WA, Kalaawry H. Laser in situ keratomileusis for myopia of -1 to -3.50 diopters. J Refract Surg 1997;13(suppl):425-6.

6. Tsai RJ-F. Laser in situ keratomileusis for myopia of -2 to -25 diopters. J Cataract Refract Surg 1997;13(suppl):427-9.

7. Salah T, Waring III GO, Maghraby AE, Moadel K, Grimm SB. Excimer laser in situ keratomileusis under a corneal flap for myopia of 2 to 20 diopters. Am J Ophthalmol 1996;121:143-55.

8. Zaldivar R, Davidorf JM, Shultz MC, Oscherow S. Laser in situ keratomileusis for low myopia and astigmatism with a scaning spot excimer laser. J Cataract Refract Surg 1997; 13:614-9.

9. Carson CA, Taylor HR. Excimer laser treatment of high and extreme myopia. Arch Ophthalmol 1995;113:431-6.

10. McCarty CA, Livingston PM, Taylor HR. Prevalence of myopia in adults: implications for refractive surgeons. J Refract Surg 1997;13:229-34. 
11. Hayashi S, Braz A, Vasques M, Plut R, Sartori M, Chammon W, Campos M. Ceratectomia fotorrefrativa com excimer laser de fluoreto de argônio de $193 \mathrm{~nm}$ para a correção da miopia: estudo clínico. Arq Bras Oftal 1997;60(2):147-51.

12. Wang Z, Chen J, Yang B. Comparison of laser in situ keratomileusis and photorefractive keratectomy to correct myopia from -1.25 to -6.00 diopters. J Refract Surg 1997;13:528-34.

13. Mori ES, Suzuki CK, Alleman N, Schor P, Campos M, Chamon W. Ceratectomia fotorrefrativa associada à ceratotomia lamelar pediculada (LASIK) para correção de miopia e astigmatismo moderados e altos em um serviço universitário. Arq Bras Oftal 1998;61(5):585-9.
14. El-Maghraby A, Salah T, Waring III GO, Klyce S, Ibrahim O. Randomized bilateral comparison of excimer laser in situ keratomileusis and photorefractive keratectomy for 2.50 to 8.00 diopters of myopia. Ophthalmol 1999;106:447-57.

15. Danasoury MAE, Maghraby AE, Klyce SD, Mehrez K. Comparison of photorefractive keratectomy with excimer laser in situ keratomileusis in correcting low myopia (from -2.00 to -5.50 diopters). Ophthalmol 1999:106:411-21.

16. Lipshitz I, Fisher L, Dotan G, Lazar M, Loewenstein A. Comparison of photorefractive keratectomy on one eye and laser in situ keratomileusis on the other eye of the same patient. J Refract Surg 1999;15(suppl):S225-S6.

\title{
XIII CONGRESSO PAN-AMERICANO
}

\section{DE}

\author{
OFTALMOLOGIA
}

22 A 25 DE JULHO DE 2.001

Hotel Sheraton - Buenos Aires - Argentina

\section{Informações: Associação Panamericana de Oftalmologia Telf.: (001-817) 265-2831 - Fax: (001-817) 275-3961 e-mail:info@paao.org}

